\title{
Bandgap and Complex Dielectric Function from the Low-Loss Energy Spectrum for SnO2 Prismatic Nano-Rods.
}

Javier Morales-Mendoza ${ }^{1}$, G. Herrera-Perez ${ }^{2}$, Carlos Ornelas-Gutiérrez ${ }^{3}$ and Francisco ParaguayDelgado $^{1}$

${ }^{1}$ Centro de Investigación en Materiales Avanzados, United States, ${ }^{2}$ Centro de Investigación en Materiales Avanzados, Chihuahua, Chihuahua, Mexico, ${ }^{3}$ Centro de Investigación en Materiales Avanzados

Tin dioxide $\left(\mathrm{SnO}_{2}\right)$ is a wide bandgap $(\mathrm{Eg}=3.6 \mathrm{eV})$ n-type semiconductor that possesses a rutile structure with a tetragonal unit cell. It is used for different technologies such as chemical sensors, optoelectronics devices, and photo-catalysis. Different synthesis methodologies are applied to prepare $\mathrm{SnO}_{2}$ such as thermal evaporation and sol-gel among others. However, the hydrothermal process is a cost-efficient methodology for the synthesis of $\mathrm{SnO}_{2}$ particles whose morphology depends on the precursor solution features. The choice of adequate parameter values is always critical to control morphology while reaching different morphologies in the micro or nanometer size range. On the other hand, electron energy loss spectroscopy (EELS) is one of the most important techniques to elucidate the local electronic structure of materials [1]. In particular, in the valence region also known as the low-loss energy region (VEELS) is possible to determine the bandgap energy Eg and the complex dielectric function. The motivation of this work is to determine the $\mathrm{Eg}$ and the complex dielectric function for $\mathrm{SnO}_{2}$ with elongated prismatic nanorods.

$\mathrm{SnO}_{2}$ was prepared by the hydrothermal method, the experimental details were reported elsewhere [2]. EELS spectra were obtained with an electron energy loss spectrometer (EELS GAT-777 STEMPack) attached to a JEM-2200FS (200 kV), which offers an energy resolution of $1.0 \mathrm{eV}$. The electron probe size was below $1 \mathrm{~nm}$. The spectra were acquired using a dispersion of $0.05 \mathrm{eV} /$ channel to record spectra up to $1000 \mathrm{eV}$. The convergence semi-angle was $\alpha=9.0 \mathrm{mrad}$ for a $2.5 \mathrm{~mm}$ spectrometer entrance aperture and $40 \mathrm{~mm}$ camera length, and the corresponding collection semi-angle was $\beta=17.3 \mathrm{mrad}$. The deconvolution of the zero-loss peak from the VEELS region, the Fourier-log method to remove plural scattering, and Kramers-Kronig analysis were carried out using the Gatan Suite software [3].

To monitor the morphology for $\mathrm{SnO}_{2}$, a high-angle annular dark-field scanning transmission electron (HAADF-STEM) micrograph was taken. The micrograph exhibits elongated prismatic nano-rods [2] and is presented in Figure 1a. Panel b shows the VEELS region where the zero-loss peak (ZLP) is identified and elemental analysis is performed for $\mathrm{SnO}_{2}$. Figure 2 a shows the ZLP that was extracted from the VEELS region considering in the fit the extrapolation of a logarithmic function [3]. The inelastic contribution spectrum was corrected for multiple scattering events using the Fourier-log method obtaining the single scattering distribution (SSD) [3]. It can be observed in this curve that the bulk plasmon is located at $21.4 \mathrm{eV}$. A linear fit up to the $\mathrm{x}$-axis in the energy loss function (ELF) suggests an $\mathrm{Eg}$ of $3.6 \mathrm{eV}$ (panel b) which agrees with the value reported in the literature [4]. Panel c exhibits the complex dielectric function, $\varepsilon 1, \varepsilon 2$, and the ELF. These curves were obtained through the Kramers-Kronig analysis [3]. VEELS-STEM profile combined with Kramers-Kronig analysis is a very useful technique to monitor (a) the electronic structure in the valence region; (b) the complex dielectric function and to determine the electronic bandgap energy Eg for rutile $\mathrm{SnO}_{2}$. 
J. M-M thanks the CONACYT Ph. D. scholarship. G. H-P thanks to CONACyT-SEP Basic Research Project No. 253605.
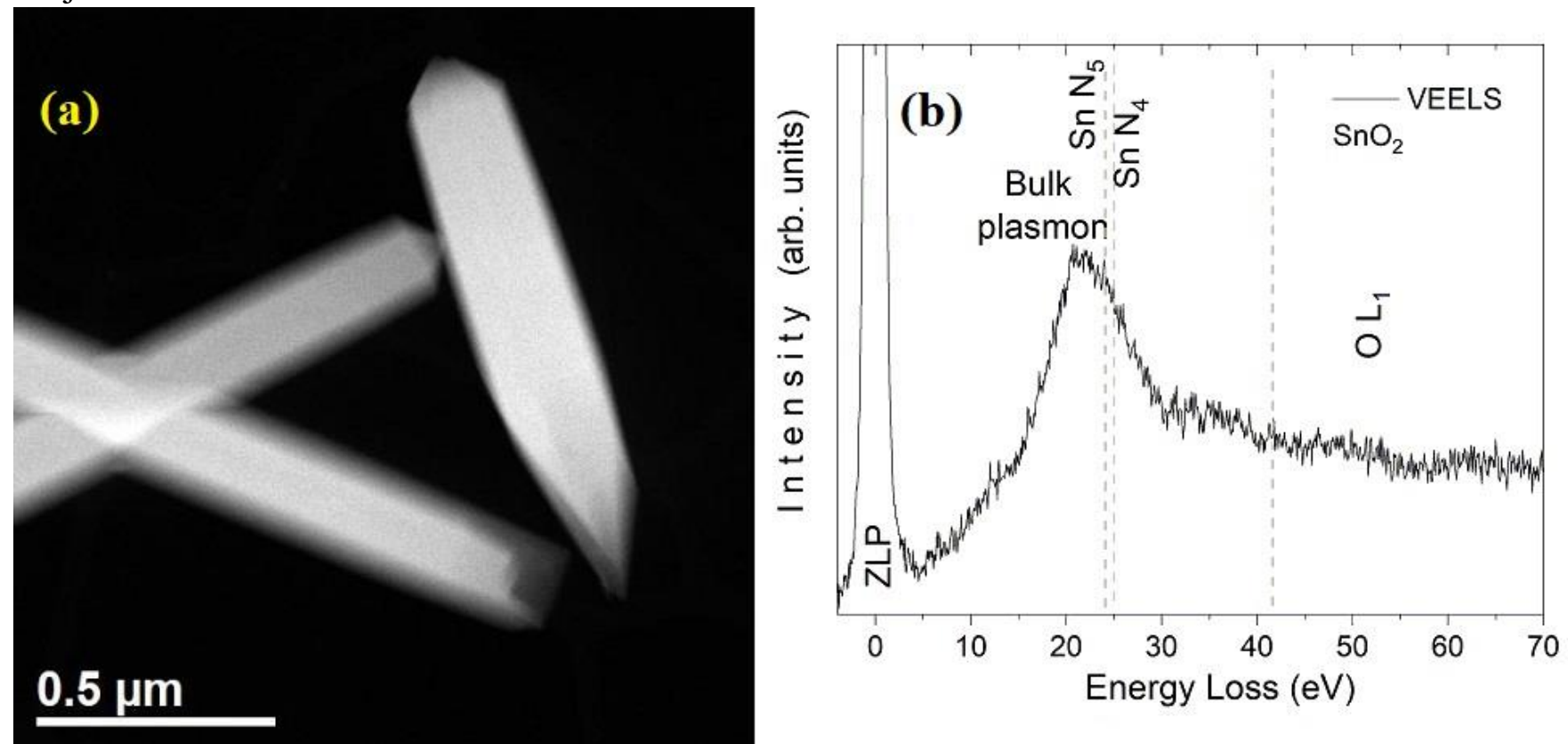

Figure 1. (a) HAADF-STEM micrograph showing elongated prismatic nano-rods. (b) Elemental identification in the VEELS region for $\mathrm{SnO}_{2}$.
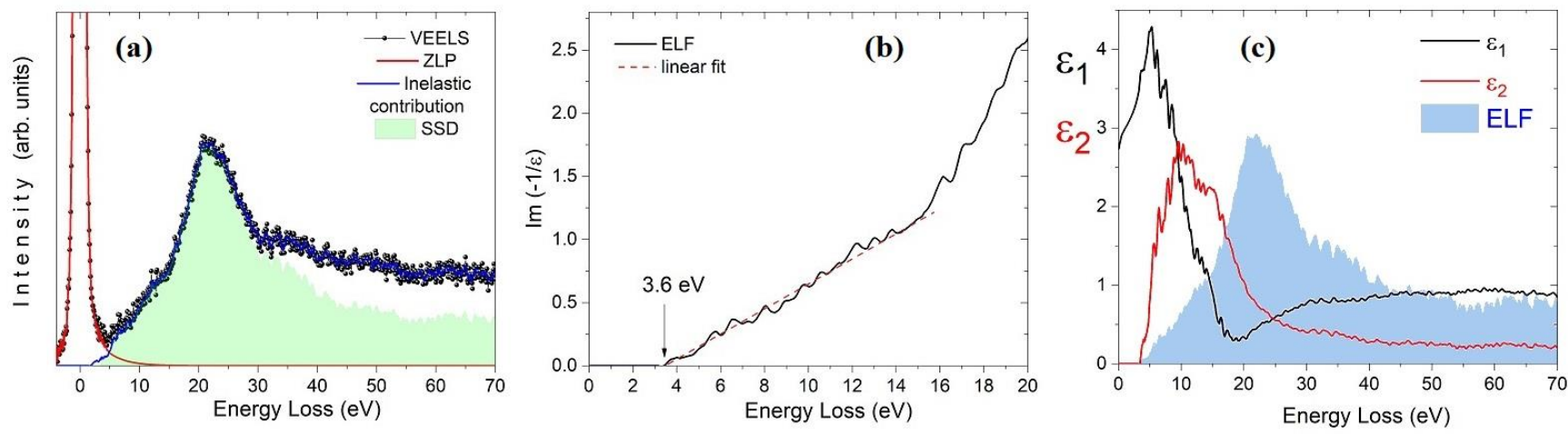

Figure 2. (a) Deconvolution of ZLP, inelastic contribution, and SSD. (b) Linear fit in the ELF to determine the bandgap energy value. (c) Complex dielectric function $\varepsilon 1, \varepsilon 2$ and ELF.

\section{References}

[1] G. Herrera-Pérez et al., J. Appl. Phys. 128 (2020), 064106.

[2] F. C. Vásquez et al., Supperlattice Microst. 90 (2016), 274-287.

[3] Digital Micrograph EELS Analysis User's Guide. Gatan, Inc. (2003) USA.

[4] D. R. Miller et al., J. Mater. Res. 32 (2017), 2479-2486 